\title{
Number of Unsuppressed Water Averages
}

National Cancer Institute

\section{Source}

National Cancer Institute. Number of Unsuppressed Water Averages. NCI Thesaurus.

Code C156495.

The integer that describes that number of averages that are captured of the unsuppressed water spectrum signal during imaging . 